\title{
Electron Beam Collector with a Low Back Flow
}

\author{
A.Shemyakin \\ Fermi National Accelerator Laboratory, Batavia, IL60510, USA \\ E-mail: shemyakin@fnal.gov, tel.(630) 840-4440, fax (630) 840-4552
}

Generation of a DC electron beam in the future Fermilab electron cooler [1] employs an electrostatic acceleration and a beam energy recovery, so that electrons are decelerated from the nominal energy of 4.3 $\mathrm{MeV}$ they have in the cooling section to few $\mathrm{keV}$ in the collector. Stable performance of this scheme requires a current loss $\delta I$ below $10 \mu \mathrm{A}$ at the beam current up to the nominal value of $I=0.5 \mathrm{~A}$. One of sources of the loss is a back flow of secondary electrons from the beam collector. The paper discusses principles and performance of a collector with the low current loss.

Electric and magnetic fields in the collectors used in existing electron coolers are axially symmetrical. For practically interesting parameters, such collectors can not provide $\delta I / I<10^{-4}$ because of the reversibility of trajectories in the collectors: a secondary electron with the kinetic energy equal to the energy of the primary one can come out of the collector following the trajectory of the "parent" electron.

The back flow can be dramatically decreased if the reversibility is broken by a transverse magnetic field in the collector cavity. In our case, the field was formed by a system of permanent magnets. Several versions of the system were tested at a low-energy test bench.

The first of them is optimised for operation without a longitudinal magnetic field. The transverse field is formed by two groups of $6 \mathrm{Nd}-\mathrm{Fe}-\mathrm{B}$ square permanent magnets (Fig.1), mounted on both sides of the collector and magnetised along the X-axis. Because the directions of magnetization in groups are opposite, the magnetic field in the vicinity of the Z-axis has a quadrupole configuration with the gradient of 10-15 G/cm. The field focuses electrons in $\mathrm{X}$ direction and defocuses in $\mathrm{Y}$, so that the beam is absorbed on collector walls mainly along a narrow band near the plane $\mathrm{X}=0$. The transverse field in this region, with the magnitude of 50-70 G, effectively confines secondary electrons. The only exception is electrons entering the collector with small Y offsets, which fly through the collector and hit its bottom. Because the transverse field strength near the bottom is low, the produced secondary electrons have a high probability of escaping from the collector.
Measurements of the collector efficiency at various beam positions at the collector entrance, made with a low-current, small size beam, show a narrow band of the beam positions with high relative current losses (up to $1 \cdot 10^{-3}$ ) near $\mathrm{Y}$ axis. At higher currents, when the beam size is comparable with the entrance opening, the beam cannot be shifted from the highloss region, and the total efficiency is determined by the beam part overlapped with the band. The best relative current loss in the symmetric configuration is $1.5 \cdot 10^{-5}$ at the beam current up to $0.5 \mathrm{~A}$.

To eliminate the effect, the magnets on one side of the collector were shifted along $\mathrm{Z}$ with respect to the second group. Arising asymmetry of the magnetic field results in a displacement of the high-loss band from the center and in decreasing of the relative losses down to $\delta I / I=3 \cdot 10^{-6}$ at the beam current of up to $I=0.6 \mathrm{~A}$ [2]. When the collector was used in a 1.2 $\mathrm{MeV}$ beam recirculation experiment at the collector voltage of $U_{c}=4 \mathrm{kV}$, the maximum beam current of $0.9 \mathrm{~A}$ and $\delta \mathrm{I} / \mathrm{I}=(5-20) \cdot 10^{-6}$ were demonstrated [3].
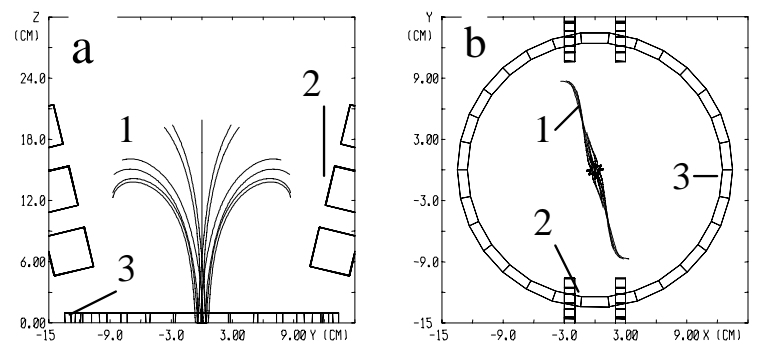

Figure 1: The simulation of electron trajectories in the equipotential space: a- YZ plane, b- XY plane. 1- electron trajectories, 2- square contours with currents of $7.3 \mathrm{kA}$ representing permanent magnets, 3- solenoid near the collector entrance. Electron energy is $5 \mathrm{keV}$, magnetic field inside the solenoid 3 is $150 \mathrm{G}$.

In the novel beam transport scheme proposed for the Fermilab cooler, the low-energy parts of the setup, including the gun and collector, should be immersed into a longitudinal magnetic field of several hundred Gauss. The field changes significantly the collector performance. First, presence of the field on the cathode introduces electron tangential velocities in the collector cavity 
and, second, the field near the collector entrance provides an additional focusing for both primary and secondary electrons. Tests of the collector described above with a solenoid placed near its entrance demonstrated a possibility to work with a higher current (up to of 1.5A) but also revealed significantly higher losses $\left(\delta \mathrm{I} / \mathrm{I}=2.5 \cdot 10^{-5}\right.$ at $\mathrm{U}_{\text {coll }}=4.2$ $\mathrm{kV}$ ). An example of simulation of electron motion made by MAG3D code [4] without taking into account the beam space charge is shown in Fig.1.

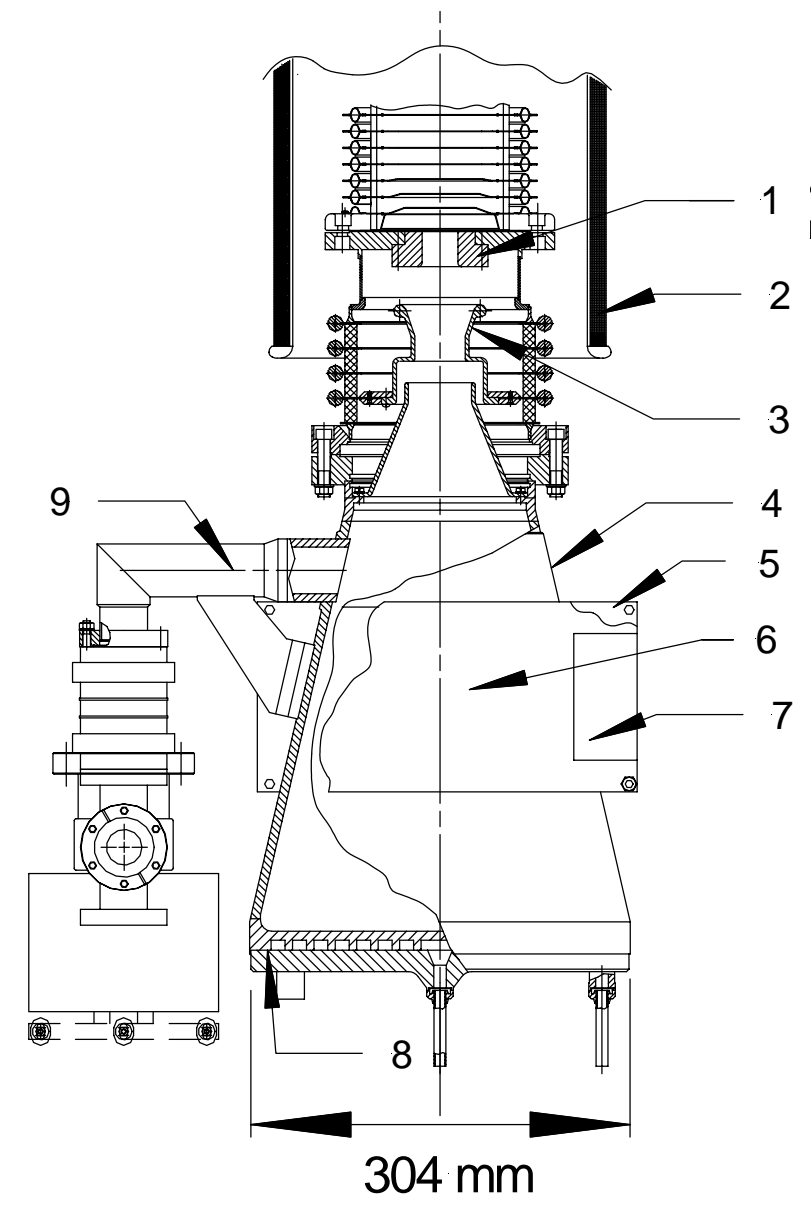

Figure 2: The collector used in the low-energy tests. 1- collector anode, 2- solenoid, 3- suppressor electrode, 4- collector body, 5 and 6- steel plates, 7permanent magnets, 8- water cooling, 9- pumping.

Much better results were found when the system shown in Fig.2 was used. After entering the collector, the beam expands to a larger diameter and is bent by a nearly homogeneous, weak transverse magnetic field. The field that strength is about $15 \mathrm{G}$ is formed by two steel plates with 1" X 2" X 4" ferrite permanent magnets attached outside each plate. The optimum field strength depends on the collector voltage and is adjusted by the quantity of magnets and by the distance between plates. In turn, the voltage is chosen according to the maximum current to be transported inside the collector cavity. Measurements shown that the current loss in this system is below $6 \mu \mathrm{A}$ at the beam currents up to 1.7 $\mathrm{A}$ at the collector voltage of $2.3 \mathrm{kV}$.

Currently the collector is used in a test facility [5] at the beam energy of $3.5 \mathrm{MeV}$ under potential with respect to the cathode of $\mathrm{U}_{\text {coll }}=4 \mathrm{kV}$. Typical dependence of the loss on the beam current is shown on Fig.3. The maximum demonstrated current is $0.75 \mathrm{~A}$ at the current loss of $20 \mu \mathrm{A}$.

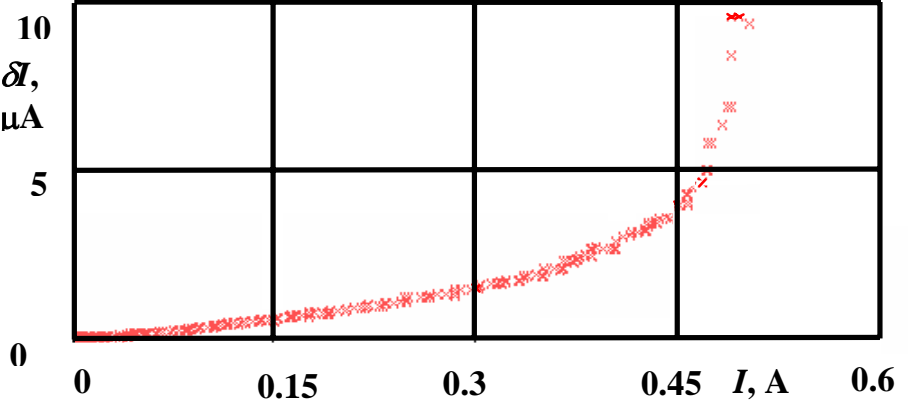

Figure 3: Current loss as a function of the beam current. The beam optics is optimised for the minimum loss at $I=0.45 \mathrm{~A}$. The magnetic field strength in the solenoid placed near the collector entrance is $200 \mathrm{G}$.

Author is thankful to the Fermilab Electron Cooling group for support and useful discussions.

\section{REFERENCES}

1. S.Nagaitsev et al., Nucl.Instr.and Meth.A441(2000) 241

2. A.Sharapa, A.Shemyakin, and S. Nagaitsev, Nucl.Instr.and Meth.-A417(1998) 177

3. A.C.Crawford et al., Nucl.Instr.and Meth.-A435 (1999), 339

4. M.A.Tiunov, B.M.Fomel,. preprint INP-83-150, Novosibirsk ,1983 (in Russian).

5. A.Burov et al., Fermilab Electron Cooling Project: commissioning of the $5 \mathrm{MeV}$ recirculation test set-up, Proc. of PAC'01, Chicago, June 18-22, 2001, http://pacwebserver.fnal.gov/papers/Wednesday/ PM_Poster/WPPH046.pdf 